\title{
Políticas de servicios sociales para la atención a personas en situación de exclusión residencial grave. Elementos para un diagnóstico y propuestas estratégicas ${ }^{1}$
}

\author{
José María Duque \\ Ayuntamiento de Bilbao \\ $<$ txema.duque@ayto.bilbao.net>
}

\begin{abstract}
Artikulu honetan, batetik, aitortzen da gizartebazterketa egoeran dauden pertsonei zuzenduriko EAEko politikek izandako arrakasta; eta, bestetik, premia azaltzen da aurrerabidea gertatzeko bizitegi-bazterketa larriari aurre egiteko gizartezerbitzuetako politiketan. Testuak kontuan hartzen ditu diru-sarbideak bermatzeko sistema, ongizate-sistemen arteko elkarkidetza eta lehenarretako gizarte-zerbitzuen gaitasun prebentiboa gizarteratzea ahalbidetzeko politika publikoen oinarrizko zutabe gisara. Administrazio, eskuartze sozialeko hirugarren sektoreko erakunde eta zientzia-komunitatearen arteko elkarkidetza oinarri harturik, egileak nabarmentzen du metodologia- eta kontzeptu-kontsentsu batean aurrerabidea egiten ari dela errealitate horren ezaguera eta azterketa hobearazteko; eta horrek garrantzia handia izango omen du Gizarte Zerbitzuen Legeak aurreikusitako Prestazio eta Zerbitzuen Katalogoa osatzeko.
\end{abstract}

\section{GAKO-HITZAK:}

Gizarte-politikak, gizarte-zerbitzuak, lehen-mailako arreta, bizitegi-bazterketa larria, etxegabeko pertsonak, zerbitzu-zorroa.
En este artículo, por un lado, se reconocen los logros en las políticas de la CAPV dirigidas a las personas en situación de exclusión; y, por otro, se plantea la necesidad de avanzar en las políticas de servicios sociales para afrontar la exclusión residencial grave. El texto considera el sistema de garantía de ingresos, la cooperación entre los sistemas del bienestar y la capacidad preventiva de los servicios sociales de atención primaria como los pilares fundamentales de las políticas públicas dirigidas a facilitar la inclusión social. A través de la cooperación entre administraciones, las entidades del tercer sector de intervención social y la comunidad científica, el autor constata que se está avanzando en la construcción de un consenso metodológico y conceptual para un mejor conocimiento y análisis de esta realidad, y que ello será de gran ayuda para el desarrollo del Catálogo de Prestaciones y Servicios previsto en la Ley de Servicios Sociales.

\section{Palabras Clave:}

Políticas sociales, servicios sociales, atención primaria, exclusión residencial grave, personas sin hogar, cartera de servicios.

${ }^{1}$ Este texto recoge, en forma de artículo, la presentación realizada por el autor en las I Jornadas sobre Exclusión Residencial en Euskadi, celebradas en Donostia-San Sebastián el 18 de marzo de 2014 (〈http://mintegia.siis.net〉). 


\section{Introducción}

En los servicios sociales, no todas las situaciones objeto de su intervención son igualmente estudiadas y, por lo tanto, conocidas. Entre las realidades más estudiadas, tenemos la situación de las personas mayores, las personas con discapacidad, la violencia de género, la desprotección de menores o la dependencia. Este interés tiene su reflejo en el desarrollo normativo, así como en la planificación y disposición de recursos y programas.

Otras realidades, como la desprotección de personas adultas y las situaciones de exclusión social, a pesar de estar contempladas en nuestras leyes de servicios sociales, no han gozado del necesario interés y preocupación para promover su conocimiento, profundización y planificación en relación a las políticas sociales y el desarrollo de los recursos adecuados a ellas. En el ámbito de la exclusión social, y concretamente respecto al conocimiento de la exclusión residencial grave (personas en situación de sinhogarismo extremo), históricamente nos hemos encontrado con dificultades derivadas de la falta de consenso conceptual, y de la movilidad física y temporal de las personas sin hogar.

En la CAPV, aunque sea de una forma muy desigual, observamos un claro aumento del interés por conocer esta realidad en los últimos años ${ }^{2}$. En particular, los ayuntamientos de Bilbao, Donostia-San Sebastián y Vitoria-Gasteiz, con la colaboración del Departamento de Empleo y Políticas Sociales y las tres Diputaciones Forales, pretenden promover no sólo el estudio, sino también el debate sobre las políticas que deben desarrollarse como respuesta a la exclusión residencial grave. Esta dinámica de cooperación institucional y, al mismo tiempo, entre administraciones, entidades del tercer sector de intervención social y comunidad científica, posibilita rigurosidad en el análisis e integración de las políticas públicas. El trabajo realizado supone una propuesta consensuada y convincente de superación de las dificultades metodológicas y conceptuales para el análisis de esta realidad.

En el contexto descrito, este artículo pretende aportar una serie de elementos que ayuden a profundizar en el diagnóstico y tratamiento de la exclusión en la CAPV, ofreciendo, a su vez, algunas propuestas estratégicas graduadas en cuanto a su alcance y concreción.

\section{Apunte conceptual}

El proceso que va desde una situación de inclusión a otra de exclusión residencial grave (y viceversa) se presenta como un continuo que, desde un punto de

${ }^{2}$ Este interés queda corroborado por la publicación de diversos estudios y artículos en torno a este tema: Comisión Onartu (2011), Deloitte (2010), Eustat (2012), Duque (2013), Duque et al. (2012), Navarro (2013), SIIS Centro de Documentación y Estudios (2011 y 2013) y Zalakain (2014). vista analítico, es necesario describir y, quizás, es posible predecir o pronosticar. El sistema de servicios sociales -al igual que otros, como el sanitario o el de garantía de ingresos- dedica gran parte de sus esfuerzos a objetivos asistenciales que ayudan a corregir los efectos de los procesos exclusógenos. Por lo tanto, nuestra capacidad para describir y analizar las causas y consecuencias (síntomas) de la exclusión incidirá proporcionalmente en las políticas preventivas $^{3}$.

\subsection{La inclusión-exclusión como situación genérica ${ }^{4}$}

Nos encontramos con una situación de inclusión social cuando se da una disponibilidad y un ejercicio de una serie de capacidades personales (competencias, habilidades, capacitación, información), sociales (apoyo social, aceptación social) y económicas. En definitiva, nos referimos al ejercicio de la ciudadanía, conformada por derechos y deberes, según el estándar de participación social de una sociedad o comunidad concreta. Por ello, consideramos que tiene un carácter multicausal y multidimensional.

La capacidad de acceder y mantener los bienes que cada sistema de protección procura a la ciudadanía (vivienda, salud, formación, empleo, renta, justicia) puede verse dificultada o limitada por diversos déficits en estas capacidades personales, sociales y económicas. En consecuencia, en la medida que una persona o familia ve disminuida la posibilidad de acceso y mantenimiento de los citados bienes, se encontrará en situación de vulnerabilidad o riesgo, y podrá llegar a una situación de privación o carencia.

Aunque la interrelación entre los distintos ámbitos carenciales sea un tema debatido, es evidente que podemos identificar situaciones de déficit o privación concernientes a cada sistema por separado. Así, nos referimos a exclusión residencial, sanitaria, económica, formativa, laboral 5 . En este sentido, una capacidad económica mermada (ligada al concepto de pobreza) condicionará, sin duda, el acceso a los demás ámbitos.

\footnotetext{
${ }^{3}$ Ante situaciones sociales complejas, algunos autores optan por hacerse grandes preguntas ('¿cuál es la causa principal de la pobreza en el mundo?'). Otros, sin embargo, defienden que es mejor analizar esa realidad como un conjunto de problemas específicos que una vez identificados y comprendidos, pueden ser resueltos de uno en uno (Banerjee y Duflo, 2012: 19 ss.).

${ }_{4}$ En este sentido se planteó en la Ley 12/1998, de 22 de mayo, contra la Exclusión Social, en cuya exposición de motivos este fenómeno se define como "la imposibilidad o la incapacidad de ejercer los derechos sociales, fundamentalmente el derecho al trabajo, pero también el acceso a la educación, a la formación, a la cultura, a la salud, a una vivienda digna, a la protección social”.

${ }^{5}$ La Aliança contra la Pobresa Energética, en Cataluña, afirma que muchas familias no tienen acceso a una vivienda digna, pero tampoco al agua, el gas y la luz (〈http://pobresaenergetica.es/১). Según la Asociación Española de Operadores Públicos de Abastecimiento y Saneamiento (AEOPAS), ya se han ejecutado más de 300.000 cortes de suministro de agua por impago (Limón, 2014). En definitiva, son exclusiones por falta de acceso a determinados bienes, como el combustible (pobreza energética), el agua (exclusión hídrica) o el ascensor (exclusión vertical).
} 
Aunque siempre ha estado presente el elemento estructural, es cierto que en estos años de crisis económica se han ido endureciendo los requisitos de acceso a los sistemas, lo que ha tenido como resultado un aumento de demanda en el sistema de servicios sociales. Como ejemplos, podemos citar el impacto de la pérdida de vivienda (desahucios) por incapacidad económica -debida, sobre todo, a la pérdida del empleo- o la pérdida de derecho a la salud por incumplimiento de requisitos para el acceso a tratamiento médico (personas extranjeras).

Por lo tanto, cuando nos encontramos con una de estas situaciones de vulnerabilidad o privación, no vamos a calificarla necesariamente de exclusión social. Aunque desde el punto de vista de su etiología pueden estar claramente interrelacionas (sobre todo con la privación económica), es importante clarificar el papel de los servicios sociales en la mejora de cada situación deficitaria relacionada con los otros sistemas. En este sentido, y sin perjuicio de la imprescindible cooperación entre los sistemas (Duque, 2012), los servicios sociales de atención primaria juegan un papel clave para facilitar el acceso al ejercicio de los derechos de la ciudadanía, al ofrecer de manera simultánea los servicios y prestaciones propias de su sistema (en especial, el acompañamiento social) [Aguilar, Llobet y Pérez Eransus, 2012].

\subsection{Exclusión residencial}

En las últimas décadas, investigadores, profesionales, organizaciones sociales y administraciones están realizando un gran esfuerzo por llegar a una definición consensuada del problema y de quiénes son las personas sin hogar. partiendo de las condiciones de alojamiento en que viven y no tanto por sus circunstancias personales. En 2005, la Federación Europea de Organizaciones Estatales que Trabajan con las Personas sin Hogar (Feantsa) formuló una tipología europea de exclusión ligada al alojamiento (ETHOS), que fue revisada en 2006 y 2007 . El citado instrumento establece cuatro categorías conceptuales en relación a tres aspectos de la situación de la vivienda (física, social y legal), y las subdivide en 13 categorías operativas, que se traducen, a su vez, en 24 situaciones residenciales. Las cuatro categorías que establece son: sin techo, sin vivienda (en alojamientos temporales, instituciones), en situación de alojamiento precario (inestable, inseguro) y en vivienda inadecuada (por estructura, fuera de legislación, infravivienda).

Así, podemos decir que una situación de exclusión residencial (que puede ser diversa, por eso ETHOS ofrece una graduación) puede ser -aunque no necesariamente-clave en un diagnóstico de exclusión social. En mi opinión, el término 'exclusión residencial grave' o 'extrema' incluye las categorías de sin techo, sin vivienda y algunas situaciones similares contempladas en la categoría denominada 'vivienda inadecuada'.
2.3. La exclusión social como categoría diagnóstica en el sistema público de servicios sociales

En el marco del derecho subjetivo establecido en la Ley $12 / 2008$ de Servicios Sociales de la CAPV, en su artículo 25 sobre los requisitos de acceso a los servicios del Catálogo (art. 22), recoge el "requisito de necesidad”, que se acreditará a través del diagnóstico social y la valoración de exclusión. Asimismo se recogen como requisitos de acceso "la idoneidad de la prestación o servicio para responder a las necesidades de la persona destinataria y la prescripción técnica” (art. 25.1.b).

El diagnóstico social y la valoración de la exclusión exploran la realidad de la persona o familia a partir de una serie de indicadores agrupados en los denominados ‘ámbitos vitales': económico-laboralresidencial, convivencial, personal, de la salud y social.

El instrumento técnico de valoración de exclusión social (Decreto 385/2013) acredita si la persona se encuentra en situación de inclusión, riesgo o exclusión, así como la intensidad de ésta, distinguiendo tres niveles: leve, moderada y grave.

\section{Desarrollo de la exclusión en el sistema público de servicios sociales}

El tratamiento de la exclusión en la CAPV participa del desarrollo general del sistema público de servicios sociales en la CAPV (Duque, 2012). En síntesis, habría que tener en cuenta los siguientes elementos:

- Desarrollo de servicios no homogéneo ni equilibrado ${ }^{6}$. No ha existido concepción de sistema único. En consecuencia, no podemos hablar de un desarrollo y despliegue homogéneo y ordenado, que responda a cierta planificación. Los dos niveles de atención -primario y secundarioresponden a dinámicas propias de dos sistemas. Contingencias como la discapacidad o la exclusión no han tenido la atención primaria como puerta de acceso al sistema. No se ha contado con herramientas homogéneas de planificación, diagnóstico y evaluación.

- Déficit en regulación de servicios y falta de consenso conceptual. La heterogeneidad y la falta de definición de requisitos funcionales, personales y prestaciones en los recursos dirigidos a la exclusión social ha dado como resultado una gran diversidad de servicios y desigualdad en ciudades y territorios históricos. Como ejemplo, tenemos conceptos muy utilizados, como baja/media/alta exigencia, grado de intensidad de apoyo profesional, centro de día, o centro de alojamiento o acogida. 
- Inadecuación en la estructura de la atención primaria. Las leyes de servicios sociales (1982 y 1996) han tenido un mínimo impacto en los servicios y programas del ámbito de la exclusión. De hecho, el acogimiento de urgencia, que la Ley de 1996 obligaba a los municipios mayores de 20.000 habitantes, lo cumplieron las tres capitales y pocos más. Los servicios sociales de base no han contado con las condiciones necesarias (orientación, herramientas, formación y terminología) para integrar la exclusión en su atención habitual. Los servicios específicos para exclusión en atención primaria se han desarrollado sin suficiente conexión con la red de servicios sociales de base.

- Traslado de la renta de garantía de ingresos (RGI) y la prestación complementaria de vivienda (PCV) al sistema de empleo. Ello se está traduciendo en una significativa recuperación de espacio y tiempo en los servicios sociales de base.

- Modificación de los requisitos y retroceso en las coberturas sociales de personas en riesgo o situación de exclusión social. En los servicios sociales de atención primaria, se percibe un aumento de personas en situación o riesgo de exclusión asociado al endurecimiento de los requisitos de acceso (tiempo de empadronamiento y limitación de rentas por vivienda).

\section{La exclusión en la CAPV en grandes cifras}

El artículo 6 de la Ley de Servicios Sociales (12/2008) establece que la finalidad del sistema vasco de servicios sociales es promover, en cooperación y coordinación con otros sistemas y políticas públicas, el bienestar social del conjunto de la población, siendo uno de sus objetivos esenciales -junto a la dependencia o autonomía personal y la desprotección- el de prevenir y atender las situaciones de exclusión, y promover la integración social de las personas, de las familias y de los grupos.

Para hacernos una idea global del alcance de la exclusión en las políticas sociales de la CAPV, tendremos en cuenta algunos indicadores en relación al gasto y la atención 7 . El sistema público de servicios sociales de la CAPV dedica aproximadamente el $54 \%$ del gasto a personas mayores y dependencia, el $20 \%$ a discapacidad y enfermedad mental, el $13 \%$ a desprotección de menores, el $6 \%$ a servicios sociales de base, el $6 \%$ a exclusión-inclusión social y el $1 \%$ a desprotección de mujer ${ }^{8}$. En el caso de las tres capi-
7 Los datos que se ofrecen a continuación son aproximados, ya que en ocasiones es difícil imputar partidas de los presupuestos a contingencias (desprotección, dependencia, exclusión) concretas. Dificultad añadida supone definir el gasto imputable a programas de prevención de la exclusión.

${ }^{8}$ Estimaciones aproximadas correspondientes a 2011. No incluyen las prestaciones económicas pertenecientes al sistema de tales, el gasto medio dedicado a exclusión supone el $18,08 \%$ del presupuesto, oscilando entre el $13 \%$ de Vitoria-Gasteiz, el 20,57\% de Bilbao y el 20,52\% de Donostia-San Sebastián.

En cuanto a los datos de atención, tenemos que subrayar de nuevo la falta de homogeneidad a la hora de recoger los datos entre las tres capitales. No obstante, podemos decir que, en 2013, Donostia-San Sebastián atendió a 2.239 personas distintas en servicios de necesidades básicas, y Bilbao, a 3.796. En ambos municipios, el porcentaje de personas atendidas por primera vez estaba aproximadamente en el $50 \%$. Por otra parte, los programas de intervención socioeducativa con personas sin hogar (que intervienen con estas personas en la calle y, más puntualmente, en centros de alojamiento, dando continuidad a la atención) atendieron a 287 personas distintas en Vitoria-Gasteiz y a 390 en Bilbao. Igualmente podemos decir que en el programa de empadronamiento a través de los servicios sociales participaron 25 personas en Vitoria-Gasteiz, 114 en Donostia-San Sebastián y 538 en Bilbao.

Además, contamos con unos datos especialmente significativos y ya conocidos a partir del estudio realizado en octubre de 2012 de manera sincronizada en las tres capitales y en todos los recursos de alojamiento temporal de competencia municipal y foral (SIIS, 2013) [Tabla 1].

Tabla 1. Incidencia y distribución de las diversas situaciones de exclusión residencial grave, por territorio histórico (CAPV, noche del 17 al 18 de octubre de 2012)

\begin{tabular}{l|c|c|c|c}
\cline { 2 - 5 } & Álava/Araba & Bizkaia & Gipuzkoa & CAPV \\
\hline $\begin{array}{l}\text { Personas localizadas } \\
\text { en calle }\end{array}$ & 29 & 148 & 66 & 243 \\
\hline $\begin{array}{l}\text { Personas ubicadas } \\
\text { en recursos con } \\
\text { alojamiento }\end{array}$ & 279 & 738 & 444 & 1.461 \\
\hline $\begin{array}{l}\text { Total personas en calle } \\
\text { y recursos }\end{array}$ & 308 & 886 & 510 & 1.704 \\
\hline $\begin{array}{l}\text { Tasa por 1.ooo } \\
\text { habitantes de personas } \\
\text { en calle }\end{array}$ & 0,12 & 0,42 & 0,35 & 0,31 \\
\hline $\begin{array}{l}\text { Tasa por 1.00o } \\
\text { habitantes de personas } \\
\text { en recurso** }\end{array}$ & 0,86 & 0,64 & 0,62 & 0,67 \\
\hline $\begin{array}{l}\text { \% de personas en calle } \\
\text { sobre el total }\end{array}$ & 9,4 & 16,7 & 12,9 & 14,3 \\
\hline $\begin{array}{l}\% \text { de personas alojadas } \\
\text { en recurso sobre el } \\
\text { total }\end{array}$ & 90,6 & 83,3 & 87,1 & 85,7 \\
\hline
\end{tabular}

* Dado que los datos se refieren exclusivamente a las personas localizadas en las capitales, esta tasa está calculada sobre esa población.

** Esta tasa se calcula sobre el total de personas residentes en cada territorio histórico.

Fuente: SIIS Centro de Documentación y Estudios (2013). garantía de ingresos (renta de garantía de ingresos, prestación complementaria de vivienda), ni las prestaciones económicas del sistema de dependencia. 


\section{Disponemos de un sistema consolidado de prestaciones económicas}

La renta de garantía de ingresos, con la prestación complementaria de vivienda, así como las ayudas de emergencia social son, desde el ámbito de la carencia económica, los instrumentos más significativos de prevención de la exclusión en la CAPV. Si atendemos al número de unidades de convivencia que han percibido la renta de garantía de ingresos (RGI) en los últimos años, podemos observar un espectacular aumento (Tabla 2).
Los datos correspondientes a Bilbao se representan en el Gráfico 1. La Tabla 3 sintetiza el gasto en ayudas de emergencia social (AES) en la CAPV y, en concreto, en las tres capitales. La disminución en 2010 se explica por el inicio en ese año de la prestación complementaria de vivienda unida a la renta de garantía de ingresos.

Además, los municipios disponen de ayudas económicas municipales no periódicas (AEMNP) que aportan de su propio presupuesto y que gestionan, en combinación con las ayudas de emergencia social, para hacer frente a situaciones de necesidad (Tabla 4).

Tabla 2. Evolución del número de número de unidades de convivencia beneficiarias de la renta de garantía de ingresos (CAPV, 2000-2013)

\begin{tabular}{l|c|c|c|c|c|c}
\cline { 2 - 6 } & $\mathbf{2 0 0 0}$ & $\mathbf{2 0 0 5}$ & $\mathbf{2 0 0 7}$ & $\mathbf{2 0 0 9}$ & $\mathbf{2 0 1 2}$ & $\mathbf{2 0 1 3}$ \\
\hline CAPV & 16.550 & 31.654 & 36.004 & 55.410 & 69.179 & 74.738 \\
\hline Araba/Álava & 2.049 & 3.958 & 4.206 & 7.641 & 9.642 & 10.768 \\
\hline Bizkaia & 10.376 & 22.239 & 25.929 & 36.839 & 45.337 & 47.513 \\
\hline Gipuzkoa & 4.125 & 5.457 & 5.869 & 10.930 & 14.200 & 16.457 \\
\hline
\end{tabular}

Fuente: Elaboración propia a partir de datos de Lanbide.

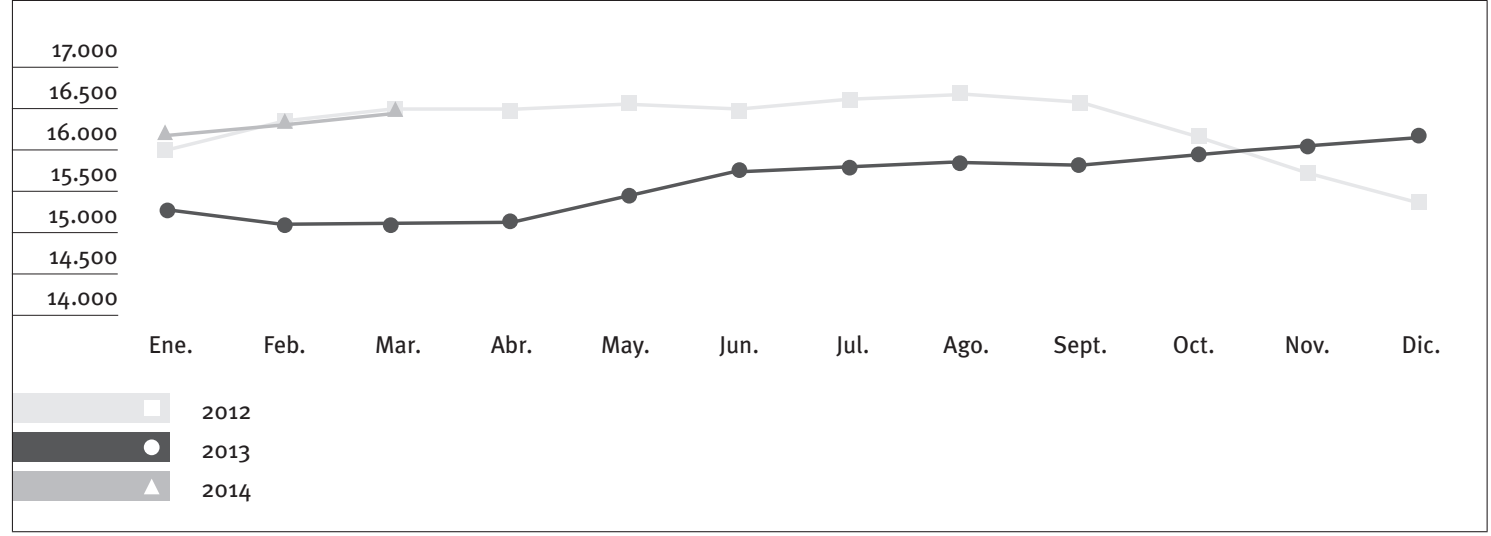

Fuente: Ayuntamiento de Bilbao a partir de datos de Lanbide.

Tabla 3. Evolución del gasto en ayudas de emergencia social (CAPV, 2007-2013, en euros)

\begin{tabular}{l|c|c|c|c|c|c|c}
\cline { 2 - 8 } & $\mathbf{2 0 0 7}$ & $\mathbf{2 0 0 8}$ & $\mathbf{2 0 0 9}$ & $\mathbf{2 0 1 0}$ & $\mathbf{2 0 1 1}$ & $\mathbf{2 0 1 2}$ & $\mathbf{2 0 1 3}$ \\
\hline CAPV & 36.000 .000 & 38.000 .000 & 54.500 .000 & 24.000 .000 & 17.300 .000 & 17.300 .000 & 19.300 .000 \\
\hline Vitoria-Gasteiz & 4.003 .781 & 4.411 .652 & 6.898 .815 & 2.458 .437 & 2.059 .372 & 2.080 .612 & 2.348 .803 \\
\hline Donostia-San Sebastián & 2.731 .728 & 2.885 .443 & 4.030 .725 & 1.719 .131 & 1.240 .085 & 1.179 .933 & 1.329 .760 \\
\hline Bilbao & 10.336 .423 & 10.991 .393 & 14.639 .695 & 5.246 .521 & 3.814 .059 & 3.866 .687 & 4.319 .023 \\
\hline
\end{tabular}

Fuente: Elaboración propia a partir de datos de la Viceconsejería de Políticas Sociales del Gobierno Vasco.

Tabla 4. Evolución del gasto en ayudas económicas municipales no periódicas (CAPV, 2007-2013, en euros)

\begin{tabular}{l|c|c|c|c|c|c|c}
\cline { 2 - 7 } & $\mathbf{2 0 0 7}$ & $\mathbf{2 0 0 8}$ & $\mathbf{2 0 0 9}$ & $\mathbf{2 0 1 0}$ & $\mathbf{2 0 1 1}$ & $\mathbf{2 0 1 2}$ & $\mathbf{2 0 1 3}$ \\
\hline Vitoria-Gasteiz & $6.784 .849,00$ & $4.424 .392,00$ & $5.472 .128,00$ & $2.283 .498,00$ & $2.955 .883,00$ & $1.426 .277,00$ & $1.426 .277,00$ \\
\hline Donostia-San Sebastián & $4.755 .066,66$ & $5.176 .599,89$ & $5.946 .270,34$ & $2.970 .440,01$ & $2.234 .163,33$ & $2.213 .479,57$ & $2.213 .479,57$ \\
\hline Bilbao & $400.000,00$ & $400.000,00$ & $400.000,00$ & $400.000,00$ & $700.000,00$ & $870.000,00$ & $1.264 .000,00$ \\
\hline Total & $11.939 .915,66$ & $10.000 .991,89$ & $11.818 .398,34$ & $5.653 .938,01$ & $5.890 .046,33$ & $4.509 .756,57$ & $4.903 .756,57$ \\
\hline
\end{tabular}

Fuente: Elaboración propia a partir de datos de la Viceconsejería de Políticas Sociales del Gobierno Vasco. 


\section{La atención primaria como espacio para la prevención}

La inmensa mayoría de las intervenciones desarrolladas en los servicios sociales de base tienen un carácter preventivo. La Ley de Servicios Sociales (12/2008), en su artículo 27.2.a, dice que los servicios sociales de atención primaria "posibilitarán el acceso de las usuarias y usuarios al conjunto del Sistema de Servicios Sociales y atenderán las necesidades relacionadas con la autonomía, la inclusión social y las situaciones de urgencia o desprotección social, con particular incidencia en la prevención de las situaciones de riesgo".

¿Cómo se llega a una situación de exclusión residencial grave? A partir de estudios realizados y de la larga experiencia de programas y centros que atienden a estas personas, conocemos con mucho detalle las causas que les han llevado a esta situación?. Las más frecuentes son las siguientes:

- Problemas económicos.

- Problemas laborales.

- Problemas o conflictos familiares o de pareja.

- Problemas con el alcohol u otras drogas.

- Problemas administrativos (papeles).

- Abandono o salida de institución (menores).

- Propia voluntad.

- Problemas de salud.

- Problemas para mantener el alojamiento.

- Problemas con la red de acogida.

- Problemas con el juego.

A modo indicativo, algunos contenidos que pueden ofrecerse en formato de programas o de intervenciones individuales o familiares en los servicios sociales de base (muchas veces, en colaboración con el sistema sanitario, educativo o vivienda) están relacionados con:

- Las personas adultas que viven solas, algunas con enfermedad mental, otras con síndrome de Diógenes, procesos de incapacitación.

- La atención en situaciones de urgencia en conflictos intrafamiliares: malos tratos, violencia de género.

- Las situaciones de exclusión grave por adicciones, consumo higiénico, reducción de daños.

- Las problemáticas personales o familiares que provocan impagos.

- Los conflictos vecinales.

- Las viviendas de inclusión: programas de vivienda social acompañada (acompañamiento social).
- La colaboración con los servicios y programas de vivienda pública (viviendas municipales, Alokabide, Bizigune).

- La colaboración con los servicios de mediación para afrontar el sobreendeudamiento por créditos hipotecarios.

- Los procesos de separaciones y divorcios.

Por lo tanto, debemos identificar, reconocer -esto es, cuantificar y mostrar-y potenciar las intervenciones, programas y servicios que están dirigidos a la detección e intervención temprana de las causas del listado anterior. Sabiendo que el sistema de servicios sociales no es suficiente para la prevención y el abordaje de todas estas cuestiones, es importante destacar la capacidad de los servicios sociales de atención primaria, que cuentan, entre sus prestaciones, con el acompañamiento social y la relación de ayuda.

\section{La exclusión en la Ley 12/2008 de Servicios Sociales}

Disponemos de una ley de servicios sociales con un marcado carácter preventivo y comunitario. En ella, se trata la exclusión en seis artículos ${ }^{10}$, y ya en la exposición de motivos, reconoce "el crecimiento de las necesidades y demandas de atención asociadas a situaciones de vulnerabilidad y de cronificación de las situaciones de exclusión, marginación y pobreza". Entre otras, se citan las dificultades de acceso a la vivienda y la precariedad laboral (mujeres, jóvenes e inmigrantes), la insuficiente protección social y las situaciones de aislamiento y soledad (personas mayores), el incremento de las situaciones de conflicto en la adolescencia y las situaciones de desprotección que afectan a personas menores de edad, como consecuencia de múltiples factores socioeconómicos y familiares.

Es probable que pronto dispongamos del Decreto de Cartera de Servicios, que regule así el Catálogo expresado en el artículo 22 de nuestra ley. Dada la importancia del citado decreto, expondré a continuación algunas preocupaciones al respecto.

\subsection{Aportación crítica al Decreto de Cartera de Servicios ${ }^{11}$}

Partimos de que el desarrollo de la Ley 12/2008 de Servicios Sociales, además de necesario, puede ayudar a ordenar y trabajar mejor, siguiendo el proceso básico de intervención establecido (ficha social, profesional de referencia, diagnóstico social, plan de atención personalizado y programación individual) y evitando las dispersión (las personas usan muchos servicios y horas de profesionales muchas veces para lo mismo -o para lo contrario, que es peor-).
9 Desde una perspectiva menos formal, véanse Bauer y Boie (2013), y Fuster (2010).

\footnotetext{
${ }^{10}$ Concretamente, en los artículos 6, 7.e, 22, 27.2.a y 46.

${ }^{11}$ Se parte del borrador de enero de 2014 .
} 
Podemos decir que el sistema de atención al ámbito de la exclusión ha avanzado mucho, en términos de recursos, profesionalización y de coordinación. Pero hay que aceptar que, en cuestiones operativas, nos movemos todavía en un cierto desorden (no tenemos los procedimientos y la claridad de otras contingencias, como dependencia y desprotección de menores) y los recursos están dispersos, siendo demasiado frecuente la dinámica del circuito asistencial, así como la difícil coordinación entre los ayuntamientos y las diputaciones forales.

Partiendo de un análisis comparado de los recursos previstos para personas en situación de riesgo y exclusión en el artículo 22 de la Ley y en el borrador de Decreto de Cartera de Servicios, ofrecemos las siguientes reflexiones que, naturalmente habrán de ser tomadas con carácter provisional, dado que parten de un borrador, y por ello, sujeto aún cambios. Las dos primeras tienen relación con aspectos conceptuales y el resto pueden ser déficits o incoherencias:

- Respecto a la naturaleza de los servicios (cfr. definición, objetivos, funciones y prestaciones). Da la impresión de que los servicios de atención primaria no se consideran dirigidos hacia un proceso de inclusión (incluso parece evitarse la expresión ${ }^{12}$ ); sin embargo, los de secundaria se definen así expresamente, incluso en los apartados 2.3.2. y 2.4.5., que se refieren a servicios para atender situaciones más cronificadas. Seguramente ello hace que el servicio de atención diurna (1.7.) no incluya la valoración de seguimiento y el acompañamiento social entre las prestaciones, y que el servicio de atención nocturna (1.8.) no incluya la orientación ni la intervención educativa.

Desde la atención primaria defendemos que no solamente ofrecemos prestaciones de necesidades básicas, sino que intervenimos en un proceso de inclusión (con diagnóstico social, plan de atención personalizado, profesional de referencia), con mejoras evidentes en el proceso de autonomía de la personas.

- En relación a la intensidad de apoyo profesional ${ }^{13}$, se establece una correspondencia directa y exclusiva entre intensidad de apoyo y el nivel de exigencia (excepto en el centro de atención nocturna 2.3.2.). Dicha intensidad de apoyo profesional no ha de estar únicamente en función del nivel de exigencia (del servicio o centro). En mi opinión, lo adecuado es combinarlas teniendo en cuenta la necesidad y capacidad de autonomía de la persona atendida. Por ejemplo, una intervención de baja intensidad (entendida como baja presencia) puede estar indicada para los primeros contactos con una persona sin hogar (atención primaria),

\footnotetext{
12 "Tendente a normalizar su situación" en 1.7. y "transición hacia una situación normalizada” en 2.2.3.

${ }^{13} \mathrm{El}$ grado de intensidad de apoyo profesional, así como el nivel de exigencia, son conceptos que, al no estar definidos, provocan confusión y mucha discusión. Avanzar en este consenso sería positivo para el sistema público de servicios sociales.
}

pero también para acompañar a personas que han alcanzado un alto grado de autonomía (atención secundaria). Esto queda expresamente excluido en el servicio vivienda tutelada (1.9.2.). Asimismo, podemos ver indicada una intensidad media-alta para apoyar un momento de crecimiento de la motivación de una persona, en la medida que aumenta el vínculo o acepta iniciar un tratamiento psiquiátrico (atención primaria) y para acompañar un proceso de rehabilitación de adicciones (atención secundaria).

Los servicios de atención primaria están condicionados principalmente por el carácter de acceso directo o la consideración de atención de urgencia, aspectos que pueden llegar a provocar desajustes importantes en el sistema de atención:

- Riesgo de exclusión: no se contempla el servicio de atención diurna ni nocturna para personas en riesgo de exclusión. Estos servicios pueden estar indicados, al menos, para las situaciones de riesgo medio y alto, pero solamente se contemplan en la vivienda tutelada (1.9.2.).

- Duración de la estancia, en el servicio de atención nocturna (1.8.), se establece una semana de estancia máxima, que puede prorrogarse hasta la valoración de la exclusión. ¿Y si no procede realizar esta valoración? ¿Y si la prescripción técnica indica una derivación a vivienda tutelada (1.9.2)? No está contemplada esta posibilidad.

La baja intensidad de apoyo, la baja exigencia y la satisfacción de necesidades básicas -en la práctica- no son coherentes con una estancia de corta duración, especialmente si se trata de personas sin motivación suficiente para acceder a servicios de atención secundaria. Estas personas necesitan atención nocturna de duración medialarga. La vivienda tutelada (1.9.2), por su parte, prevé una estancia de duración corta-media, pero para muchas personas no será suficiente. Habrá que articular programas de acceso a vivienda con acompañamiento.

- Conciencia de situación y motivación de cambio: en el ámbito de la exclusión, ambas cuestiones están muy relacionadas con el momento (oportunidad) y circunstancias de la situación vital de la persona. Una valoración (2.1.) diferida en el tiempo difícilmente se va a corresponder con el estado de motivación detectado en la atención primaria. Por otro lado, la atención primaria se ve abocada a trabajar o apoyar la motivación de las personas con baja intensidad de apoyo profesional.

- Rechazar tratamiento psiquiátrico (enfermedad mental): esta circunstancia se presenta unida a la enfermedad infecto-contagiosa en el requisito administrativo (excepto en el servicio de atención nocturna 1.8.). En la práctica, será muy difícil de gestionar, porque hay muchas personas con alteraciones mentales sin diagnóstico psiquiátrico, además de las personas diagnosticadas sin adhesión a tratamiento. 
- Confusión en la prescripción de servicio de atención nocturna (1.8.) y de centro de atención nocturna (2.3.2.). Las denominadas 'especiales necesidades o circunstancias' no queda claro si son o no suficientes para prescribir el servicio 2.3.2. o el 2.4.5., o si es preciso que el servicio de atención nocturna (1.8.) "no pueda responder a las especiales necesidades". Por otro lado, en el caso del servicio 2.4.5., ¿cómo se compagina con su requisito de necesidad 'conciencia y motivación’?

\section{Evolución en la atención: los recursos han ido mejorando en cantidad y calidad, principalmente en las tres capitales}

La evolución de la naturaleza y objetivos de los programas ha girado fundamentalmente en torno a dos ejes: primero, la intervención social entendida no sólo como prestación asistencial (alojamiento, alimento, vestido), sino orientada hacia procesos integrales de inclusión social; y segundo, la progresiva consideración de las estrategias de reducción de riesgos -en muchos casos, imprescindibles y finalistascomo objetivos en los procesos de incorporación. Por otro lado, si nos fijamos en la cantidad y tipología de los recursos, observamos que el avance viene marcado por el aumento de la capacidad de atención (aumento de profesionales, plazas y prestaciones), así como por la diversificación de los programas.

Hay que destacar las experiencias de coordinación que, en este ámbito, se han ido sucediendo en los últimos años. El esfuerzo y la disponibilidad mostrada por profesionales, instituciones y organizaciones públicas y privadas suponen, en este momento, un capital clave para continuar con el desarrollo conseguido. Asimismo, se han ido realizando diferentes experiencias de coordinación entre los servicios sociales municipales con otras áreas municipales, así como con otros sistemas del bienestar, especialmente con el sistema de salud, el de empleo, el judicial y el de vivienda. Todo ello parte de la idea de que el abordaje de la exclusión no es materia exclusiva de los servicios sociales, sino que es imprescindible el concurso de los demás sistemas.

Este claro proceso de mejora se ve condicionado por dos situaciones: el crecimiento en plazas de alojamiento temporal y el efecto embudo en los servicios residenciales de atención primaria.

\section{a. Crecimiento en plazas de alojamiento temporal}

¿Alojamiento temporal o vivienda? Para lograr una adecuada prescripción de recursos, el alojamiento temporal está indicado cuando nos encontramos ante un diagnóstico de necesidad temporal. Éste se da en procesos de rehabilitación, crisis personales o situaciones de emergencia. Nos referimos a situaciones en que la persona no es autónoma y necesita un tutelaje de carácter temporal. Sin embargo, no tiene sentido si pretendemos cubrir una necesidad crónica o permanente de alojamiento.

Tanto desde la perspectiva de la prevención como de la asistencia, el horizonte está en la vivienda y no tanto en el alojamiento temporal. En este sentido, y en el actual contexto de crisis económica duradera, las prestaciones económicas públicas -renta de garantía de ingresos (RGI), prestación complementaria de vivienda (PCV) y ayudas de emergencia social (AES)- son el instrumento más importante de prevención de sinhogarismo en la CAPV.

La vivienda pública, por sus precios más accesibles, adquiere un significado especial para las personas no incorporadas (o sólo parcialmente) al mercado laboral. Por ello, se hace absolutamente necesaria la colaboración entre las administraciones con el objeto de establecer estrategias y programas que faciliten el acceso a la vivienda de las personas que, por discapacidad funcional 0 limitaciones para realizar actividades para la vida diaria (AVD), nunca podrán acceder a ella, a no ser que se apoyen en medidas de discriminación positiva.

b. Efecto embudo en los servicios residenciales de atención primaria

El índice de rotación en los recursos de alojamiento temporal está seriamente limitado por dos cuestiones: por un lado, la tardanza en el acceso a recursos residenciales para continuar procesos de rehabilitación o recuperación (especialmente en enfermedad mental); $y$ en segundo lugar, la falta de alternativas para el acceso a la vivienda (social y libre) y programas que incluyen acompañamiento social (permanente o temporal).

Hace ya algunos años que tenemos detectado este problema en el acceso a los servicios residenciales de atención secundaria. En el momento actual ${ }^{14}$, como efecto de la mejora en la atención a personas con enfermedad mental ( $y$ en lo relativo a detección y tratamiento), este efecto embudo es una realidad que se hace más evidente. Por lo tanto, el problema se ha trasladado a la salida de la etapa encargada a la atención primaria, y es claramente necesario aumentar las alternativas de alojamiento de media y larga estancia para personas con trastorno mental.

Respecto al avance experimentado en el ámbito de la atención, podemos concluir que es conveniente plantearse una apuesta por la vivienda, con o sin acompañamiento social ${ }^{15}$.

${ }^{14}$ A ello está contribuyendo, sin duda, los avances en la cooperación entre el sistema sanitario y el de servicios sociales.

15 En este sentido, sería interesante realizar algún estudio que nos permitiera cuantificar la demanda real, planteando una hipótesis concreta que incluyera, al menos, aspectos como número de plazas, número de profesionales e indicadores de atención y evaluación. 


\section{Conocimiento de la realidad, intervención y evaluación}

Volviendo al aumento del interés que se observa en la CAPV en los últimos años por el tema que nos ocupa, actualmente disponemos de una serie de valiosos estudios y análisis sobre la realidad de las personas en situación de exclusión residencial grave que, en mi opinión, nos ofrecen algunos resultados significativos:

- Se están dando importantes avances en cuanto al consenso metodológico y conceptual, partiendo de la clasificación ETHOS ${ }^{16}$ (Feantsa, 2005), en recuentos o peinados nocturnos (noches $S)^{17} \mathrm{y}$ utilizando cuestionarios homogéneos en los distintos momentos de las mediciones, lo cual nos permite disponer de series comparables.

- Contamos con una más que aceptable cantidad y calidad de datos en relación a lugares (mapa temático), recursos y situación de las personas.

- Hay un elevado nivel de participación social, concretado en la colaboración entre las administraciones, las entidades del tercer sector, la ciudadanía y la comunidad científica.

- Una mayor y más adecuada visibilización de esta realidad supone avanzar en concienciación y legitimación social de las políticas dirigidas a prevenir y paliar los efectos de la exclusión residencial grave.

Habida cuenta del avance anteriormente expuesto, es preciso añadir alguna propuesta que pueda ser abordada a corto plazo. Por un lado, sería muy interesante dar carácter periódico a los estudios y jornadas técnicas. Sería deseable también que se sumaran a ellos los ayuntamientos de tamaño medio de la CAPV. Por otro lado, es conveniente acompasar los procesos y la metodología de estudio con las recogidas de datos realizadas por el INE y Eustat. Además, es preciso hacer un esfuerzo para que el sistema de atención (diagnóstico, prescripción, derivación, plan de atención personalizado, profesional de referencia) garantice una coordinación formal y una continuidad de la atención. Asimismo debemos seguir avanzando en la homogeneización del registro y la recogida de datos relacionados con el ámbito de la intervención ${ }^{18}$.

${ }^{16}$ A este respecto, es preciso valorar la inclusión de la clasificación ETHOS en el Manual de Diagnóstico Social, instrumento común para la CAPV (Decreto 353/2013).

${ }^{17}$ En la CAPV, Bilbao (Deloitte, 2010; Comisión Onartu, 2011) y Donostia-San Sebastián (SIIS Centro de Documentación y Estudios, 2011) han sido escenario de este tipo de estudios; además, en 2012 se realizó una investigación simultánea en las tres capitales vascas (SIIS Centro de Documentación y Estudios, 2013). En el resto del Estado, se han llevado a cabo recuentos nocturnos en Barcelona, (Cabrera, Rubio y Blasco, 2008; Sales, 2012), Zaragoza (Cabrera, 2010) y Madrid (Muñoz et al., 2012).

${ }^{18}$ En este aspecto tenemos un gran camino por hacer en la CAPV. La Ficha Social, el Diagnóstico Social (Decreto 353/2012), y el Instrumento de Valoración de la Exclusión (Decreto 385/2013) nos ayudarán a objetivar, pero es preciso continuar con los planes de atención personalizados, y las prestaciones técnicas, materiales o económicas, por ejemplo.
Además, nos encontramos con un gran déficit en evaluación. Podemos decir que es una de las grandes tareas pendientes. Evaluar la prevención en exclusión es difícil, pero al menos podemos avanzar en identificar lo que hacemos, reconocerlo y analizarlo. Evaluar la atención precisa tener identificados los objetivos (diversos grados) que pretendemos conseguir: ¿qué pasa con las personas después de ser atendidas?, ¿continúan procesos satisfactorios?, ¿fracasan?, ¿son intervenciones puntuales (con valor en sí mismas)? Podríamos preguntarnos en qué medida estamos en un sistema de puerta giratoria.

La evaluación también está relacionada con la sensibilización y legitimación social de los servicios sociales. La ciudadanía debe conocer lo que hacemos, los logros, las dificultades, el gasto. Trabajar el mensaje de que se trata de una inversión en términos de prevención y atención que tiene una gran incidencia en ahorro para otros sistemas (sanitario, justicia, policial). Para que una realidad se reconozca, es preciso nombrarla.

\section{Líneas estratégicas en el ámbito de a exclusión}

Una vez tratados los principales logros conseguidos y sus déficits, veamos algunos retos que, en mi opinión, debemos plantearnos ${ }^{19}$ :

- Cooperación entre los sistemas de servicios sociales, vivienda, salud y de empleo (garantía de ingresos), sin olvidar el judicial. Se trataría de dar un salto cualitativo que posibilite realmente entrelazar sus políticas, de avanzar en una cooperación eficaz (más allá del buen entendimiento entre profesionales), operativa, burocratizada lo justo y duradera. Con objeto de detectar y actuar lo antes posible y así prevenir el agravamiento de las situaciones de exclusión, será preciso planificar adecuadamente la cooperación con otros sistemas, especialmente con el sanitario, el de vivienda, el judicial y el laboral.

- Modelo de atención de exclusión en la CAPV. Más allá de la Cartera de Servicios, es conveniente:

- Profundizar en el procedimiento básico de intervención en la atención primaria (diagnóstico social, plan de atención personalizada - programación individual, profesional de referencia).

- Establecer el procedimiento de intervención en exclusión entre ayuntamientos y diputaciones.

- Sistematizar los procesos de evaluación (es preciso confirmar, o no, la hipótesis de recurrencia en el circuito asistencial).

19 Sobre esta cuestión, puede verse también Aguilar, Llobet Pérez Eransus (2012); Comisión Europea y Feantsa (2010); Faciam (2010); Fresno, Renes y Tsolakis (2012); Gobierno Vasco (2012); Ministerio de Sanidad, Política Social e Igualdad (2011); y Navarro (2013). 
- Potenciación de la atención primaria con carácter preventivo y asistencial. Se trata de no llegar a la pérdida de la vivienda. A partir de los decretos de Ficha Social y Diagnóstico Social (353/2012), así como el de Valoración de la Exclusión (385/2013), nos situamos en un nuevo escenario en el que es evidente la necesidad de:

- Servicios de intervención socioeducativa más diversificados.

- Servicios de atención diurna donde las personas satisfagan necesidades básicas y avancen en su inclusión social.

- Servicios de atención nocturna.

- Viviendas tuteladas o fórmulas similares.

- Programas de acceso y mantenimiento de la vivienda, quizás unidos a intervención socioeducativa.

Desde la perspectiva de los servicios, propongo prestar una especial atención a:

- La intervención socioeducativa con adultos. La intervención socioeducativa (no solamente ligada a familias con menores) es un instrumento fundamental en una estrategia preventiva del sinhogarismo en el ámbito de los servicios sociales de atención primaria. Desde este servicio, pueden articularse los programas de acceso a la vivienda y mantenimiento permanente (o al menos, a largo plazo) de ésta.
- Los servicios de apoyo a la emancipación de menores y jóvenes extranjeros no acompañados. La salida de menores de centros de las diputaciones forales en el momento que se considera que han cumplido la mayoría de edad es una de las causas más frecuente de sinhogarismo en la CAPV. Potenciar los programas de emancipación con apoyo profesional disminuirá mucho el riesgo de estas personas.

- Servicios residenciales con apoyo para personas con enfermedad mental. Como resultado de la mejora de la cooperación entre los servicios sociales y el sistema sanitario, especialmente con la Red de Salud Mental de Bizkaia (RSMB) y, concretamente, con el Programa de Asistencia Psiquiátrica a Personas Sin Hogar con Enfermedad Mental Grave (Duque et al., 2012), hemos experimentado un aumento considerable de personas que se encuentran en procesos evidentes de mejora e incorporación social. Superados los primeros meses de adherencia al tratamiento y normalización social, muchas de estas personas se mantienen en albergues por falta de plazas específicas de larga estancia con acompañamiento social para continuar sus procesos de inserción.

- Empadronamiento a través de los servicios sociales. Supone el acceso a una serie de derechos relacionados con la inclusión y el bienestar. En el ejercicio de la responsabilidad municipal, es preciso que este programa se extienda a todos los municipios que lo puedan necesitar. 
(2014): “Decreto Foral 5/2014, de 4 de marzo, por el que se regula el procedimiento de acceso a los recursos de inclusión social de atención secundaria dependientes de la Diputación Foral de Gipuzkoa", Boletín Oficial de Gipuzkoa, nํ5 52, 18-3-2014 [shttps://ssl4.gipuzkoa.net/ castell/bog/2014/03/18/c1402536.pdf >].

(2014): "Decreto de Cartera de Servicios" [documento de trabajo, inédito], Vitoria-Gasteiz, Eusko Jaurlaritza-Gobierno Vasco, enero.

(2013): "Decreto 385/2013, de 16 de julio, por el que se aprueba el Instrumento de Valoración de la Exclusión Social”, Boletín Oficial del País Vasco, no 149, 7-8-2013 [<http://www.lehendakaritza. ejgv.euskadi.net/r48-bopv2/es/bopv2/ datos/2013/08/1303530a.pdf >].

(2013): "Decreto 353/2013, de 28 de mayo, de Ficha Social del Sistema Vasco de Servicios Sociales y del Instrumento de Diagnóstico Social del Sistema Vasco de Servicios Sociales", Boletín Oficial del País Vasco, no 121, 26-6-2013 [ ['http:// www.lehendakaritza.ejgv.euskadi.net/r48bopv2/es/bopv2/datos/2013/06/1302907a. $p d f>]$.

(2008): "Ley 12/2008, de 5 de diciembre, de Servicios Sociales", Boletín Oficial del País Vasco, no 246, 24-12-2008, págs. 31.840-31.924 [<http://www.euskadi.net/bopv2/ datos/2008/12/0807143a.pdf>].

(1998): “Ley 12/1998, de 22 de mayo, contra la exclusión social”, Boletín Oficial del País Vasco, n- 105 , 8-6-1998, págs. 10.467-10.506 [rhttp://www. lehendakaritza.ejgv.euskadi.net/r48-bopv2/ es/bopv2/datos/2007/07/0703900a.pdf〉]

(1996): "Ley 5/1996, de 18 de octubre, de Servicios Sociales”, Boletín Oficial del País Vasco, no 218,
12-11-1996, págs. 17.578-17.599 [<http://www. lehendakaritza.ejgv.euskadi.net/r48-bopv2/ es/bopv2/datos/1996/11/9605350a.pdf)].

(1982): "Ley 6/1982, de 18 de mayo, sobre 'Servicios Sociales"', Boletín Oficial del País Vasco, no 71, 2-6-1982, págs. 1.263-1.273 [<http://www. lehendakaritza.ejgv.euskadi.net/r48-bopv2/ es/bopv2/datos/1982/06/8200685a.pdf)].

AGUILAR, M.; LLOBET, M.; y PÉREZ ERANSUS, B. (2012): "Los servicios sociales frente a la exclusión", Zerbitzuan, no 51 , págs. 9-26 [<http://dx.doi. org/10.5569/1134-7147/51.01>].

ALIANÇA CONTRA LA POBRESA ENERGÉTICA (s/d) [<http:// pobresaenergetica.es〉].

BANERJEE, A.; y DUFLO, E. (2012): Repensar la pobreza. Un giro radical en la lucha contra la desigualdad global, Madrid, Taurus.

BAUER, J.; BOIE, K. (2013): Una vida cualquiera, Lóguez Ediciones, Salamanca.

CABRERA, P. J. (2010): Estudio sobre las personas sin techo en Zaragoza, Zaragoza, Cruz Roja Española.

CABRERA, P.; RUBIO, M. J.; y BLASCO, J. (2008): ¿Quién duerme en la calle? Una investigación social y ciudadana sobre las personas sin techo, Barcelona, Fundació Caixa Catalunya.

COMISIÓN EUROPEA; y FEANTSA (2010): Conferencia de Consenso Europea sobre las Personas sin Hogar, Bruselas, 9 y 10 de diciembre.

COMISIÓN ONARTU (2011): Informe técnico sobre la situación de las personas sin hogary los recursos de ámbito municipal y foral, Bilbao, Ayuntamiento de Bilbao.

DELOITTE (2010): Análisis de situación de las personas en situación de exclusión residencial grave, 
Bilbao, Grupo de Trabajo Onartu; Bilbao, Ayuntamiento de Bilbao.

DUQUE, J. M. (2013): “Investigación sobre exclusión residencial grave en la CAPV”, en OLALDE, A.; y LÓPEZ RUIZ DE AZÚA, I. (coords.), Investigación y trabajo social: dialogando desde la intervención, Universidad del País Vasco, págs. 143-162.

- (2012): “Redescubrimiento de los servicios sociales de atención primaria: hacia un (nuevo) modelo de atención personal y comunitaria", Zerbitzuan, nํ52, págs. $23-44$ [<http://dx.doi. org/10.5569/1134-7147.52.02>].

DUQUE, J. M. et al. (2012): “Evolución del Programa de Asistencia Psiquiátrica a Personas Sin Hogar con Enfermedad Mental Grave en el municipio de Bilbao", Zerbitzuan, nํㅜ 52, págs. 179-190 [<http://dx.doi.org/10.5569/1134-7147.52.12)].

EUSTAT (2012): Encuesta sobre las Personas sin Hogar 2012, Vitoria-Gasteiz, Eustat.

FACIAM (2010): 10 Propuestas para una estrategia de erradicación del sinhogarismo, Federación de Asociaciones de Centros para la Integración y Ayuda de Marginados.

FEANTSA (2005): ETHOS - European Typology of Homelessness and Housing Exclusion, Bruselas, Federación Europea de Organizaciones Estatales que Trabajan con las Personas sin Hogar [<http://www.feantsa.org/ spip.php?article120\&lang=en〉].

FRESNO, J. M.; RENES, V.; y TSOLAKIS, A. (2012): "Estrategia Europa 2020 e inclusión social: distanciamiento creciente entre objetivos, políticas e instrumentos", Zerbitzuan, $\mathrm{n} \times 51$, págs. 27-47 [ [http://dx.doi.org/10.5569/11347147/51.02)].

FUSTER, M. (2010): Miguel. 15 años en la calle, Glenat, Barcelona.

GOBIERNO VASCO (2012): III Plan de Inclusión (2012-2016), Vitoria-Gasteiz, Eusko Jaurlaritza-Gobierno Vasco [rhttp://www.gizartelan.ejgv.euskadi. net/r45-continns/es/contenidos/informacion/ actuaciones_documentacion/es_documen/ adjuntos/plan_inclusion.pdf $>$ ].

LIMÓN, R. (2014): “Los cortes de agua por impago se disparan con la crisis",
El País, 17-2-2014 [khttp://sociedad. elpais.com/sociedad/2014/02/17/ actualidad/1392670324_651915.html/].

MINISTERIO DE SANIDAD, POLÍTICA SOCIAL E IGUALDAD (2011): Configuración de una red local de atención a personas sin hogar integrada en el sistema público de servicios sociales. 100 Argumentos y propuestas, serie: Informes, Estudios e Investigación, Madrid, Ministerio de Sanidad, Política Social e Igualdad.

MUÑOZ, M. et al. (dirs.) (2012): Operación de recuento nocturno de personas viviendo sin techo en las calles de Madrid. 13 de diciembre de 2012. Principales resultados, Madrid, Ayuntamiento de Madrid.

NAVARRO, M. A. (2013): “El fin del sinhogarismo en Euskadi ¿mito o realidad?", Zerbitzuan, $\mathrm{n}^{0}$ 54, págs. 111-125 [khttp://dx.doi.org/10.5569/11347147.54.07〉].

SALES, A. (2012): La situació de les persones sense llar a Barcelona el 8 de novembre de 2011 i l'evolució dels serveis residencials, Barcelona, Xarxa d'Atenció a Persones sense Llar de Barcelona.

SIIS CENTRO DE DOCUMENTACIÓN Y ESTUDIOS (2013): Estudio sobre la situación de las personas en situación de exclusión residencial grave en la CAPV, Vitoria-Gasteiz, Eusko JaurlaritzaGobierno Vasco [<http://www.siis.net/es/ descarga-informes.php?id=257>].

- (2011): Kale Gorrian. Estudio sobre la situación de las personas sin hogar en Donostia-San Sebastián / Kale Gorrian. Donostian etxerik gabeko pertsonen egoerari buruzko azterlana, Donostia-San Sebastián, Ayuntamiento de Donostia-San Sebastián; Donostia-San Sebastián, Kale Gorrian [<http://www.siis.net/ es/ver-detalle. php? $r$ ref=201365'].

ZALAKAIN, J. (2014): “Bizitegi-bazterketa Euskadin: kuantifikazioa, ezaugarritzea eta baliabidemapa / Exclusión residencial en Euskadi: cuantificación, caracterización y_mapa de recursos" [presentación], I Jornadas sobre Exclusión Residencial en Euskadi, Donostia-San Sebastián, 18-2-2014 [khttp://mintegia.siis. net/files/presentaciones/joseba_zalakain. pptx>]. 